\title{
Biomechanical impairments and gait adaptations post-stroke: Multi-factorial associations
}

\author{
Theresa Hayes Cruz ${ }^{a, b}$, Michael D. Lewek ${ }^{c}$, and Yasin Y. Dhaher ${ }^{a, b},{ }^{*}$ \\ a Northwestern University Department of Biomedical Engineering, 345 East Superior Street, \\ Chicago, IL 60611, United States \\ b Rehabilitation Institute of Chicago, Sensory Motor Performance Program, 345 East Superior \\ Street, Chicago, IL 60611, United States \\ c University of North Carolina, Division of Physical Therapy, 3043 Bondurant Hall, CB\#7135 \\ Chapel Hill, NC 27599, United States
}

\begin{abstract}
Understanding the potential causes of both reduced gait speed and compensatory frontal plane kinematics during walking in individuals post-stroke may be useful in developing effective rehabilitation strategies. Multiple linear regression analysis was used to select the combination of paretic limb impairments (frontal and sagittal plane hip strength, sagittal plane knee and ankle strength, and multi-joint knee/hip torque coupling) which best estimate gait speed and compensatory pelvic obliquity velocities at toeoff. Compensatory behaviors were defined as deviations from control subjects' values. The gait speed model $(n=18 ; p=0.003)$ revealed that greater hip abduction strength and multi-joint coupling of sagittal plane knee and frontal plane hip torques were associated with decreased velocity; however, gait speed was positively associated with paretic hip extension strength. Multi-joint coupling was the most influential predictor of gait speed. The second model $(n=15 ; p<0.001)$ revealed that multi-joint coupling was associated with increased compensatory pelvic movement at toeoff; while hip extension and flexion and knee flexion strength were associated with reduced frontal plane pelvic compensations. In this case, hip extension strength had the greatest influence on pelvic behavior. The analyses revealed that different yet overlapping sets of single joint strength and multi-joint coupling measures were associated with gait speed and compensatory pelvic behavior during walking post-stroke. These findings provide insight regarding the potential impact of targeted rehabilitation paradigms on improving speed and compensatory kinematics following stroke.
\end{abstract}

\section{Keywords}

Strength; Torque coupling; Gait; Pelvis

\section{Introduction}

Disturbances in lower limb movement following stroke can be attributed to damaged descending neural pathways, resulting in weakness, exaggerated reflex activity, and

\footnotetext{
(C) 2009 Elsevier Ltd. All rights reserved.

* Corresponding author at: Rehabilitation Institute of Chicago, Sensory Motor Performance Program, 345 East Superior Street, Chicago, IL 60611, United States. Tel.: +1312 238 1408; fax: +1312 238 2208. y-dhaher@ northwestern.edu (Y.Y. Dhaher)..

Conflicts of interest statement

The authors and collaborators have no conflicts of interest.
} 
impaired coordination. Many previous investigations to understand the causes of gait abnormalities by examining relationships between functional activity and single joint motor impairments are equivocal. For example, gait speed has been associated with a myriad of single joint factors, including hip flexion (Chen et al., 2005), knee extension (Bohannon and Andrews, 1990; Hsu et al., 2003), ankle plantarflexion (Nadeau et al., 1999b), and ankle dorsiflexion strength (Lin et al., 2006). With few exceptions (Kim and Eng, 2003; Lin et al., 2006), the relative effect of each contributing factor has yet to be explored. These studies highlight the lack of consensus as to the primary volitional motor impairments underlying gait abnormalities post-stroke.

In the aforementioned studies, researchers investigated associations between single joint sagittal plane strength metrics and gait velocity, which is the result of movement by a multidegree-of-freedom system. Moreover, associations between gait velocity and sagittal plane strength may provide limited information, as individuals with stroke commonly exhibit abnormal movements in the frontal and transverse planes during swing phase (Perry, 1992). These compensatory movements, including circumduction and hip hiking (Perry, 1992), suggest that frontal plane strength may influence gait behaviors. Furthermore, we argue that abnormal multi-joint coupling (e.g., across-joint torque coupling (Cruz and Dhaher, 2008)) would strongly influence the impaired locomotor patterns post-stroke. The inclusion of multi-joint torque coupling in models of gait dysfunction is a necessary step to assess the effect of impaired motor coordination on locomotor performance.

The goal of this work is to develop multiple linear regression models to explore the potential influence of single joint strength and multi-joint torque coupling on gait outcomes.

Specifically, self-selected gait speed and frontal plane hip and pelvis kinematics will be used as metrics of gait performance and compensatory movements, respectively. These gait measures were related to strength and coordination metrics assessed in a biomechanically relevant, standing posture that mimics the initiation of swing. Delineating the relative effects of impaired strength and across-joint coordination on gait may facilitate the design of targeted interventions to improve ambulation post-stroke.

\section{Methods}

Subjects were recruited from the Rehabilitation Institute of Chicago's Clinical Neuroscience Research Registry to perform three testing sessions (a gait analysis, a seated assessment (isometric knee and ankle strength), and a standing assessment (isometric hip strength and multi-joint coupling)). Inclusion criteria for the Stroke group included: (1) clinical symptoms consistent with a single ischemic or hemorrhagic unilateral cortical brain lesion, confirmed by an imaging study, resulting in sensory motor dysfunction more than 12 months prior to evaluation, and (2) the ability to walk $10 \mathrm{~m}$ without assistance at a of speed $>0.4 \mathrm{~m} / \mathrm{s}$ with or without an assistive device, indicative of community ambulators with high limitations (Perry et al., 1995). Exclusion criteria included severe osteoporosis, cardiorespiratory or metabolic diseases, unhealed decubiti, a history of balance deficits not related to the stroke, persistent infection, and significant cognitive or communication impairments which would prevent understanding of study procedures. Control subjects of similar mean age, weight, and height with no prior neurological or orthopedic conditions were also recruited. Experimental procedures were approved by the Institutional Review Board of Northwestern University and written informed consent was obtained.

Overground gait analysis was performed using a passive eight camera motion analysis system (Motion Analysis Corp, Santa Rosa, CA, $120 \mathrm{~Hz}$ ). Retroreflective markers were taped to the posterior sacrum, the bilateral anterior superior iliac spines (ASIS), medial and lateral femoral condyles, medial and lateral malleoli, posterior heel counter of the shoe, and 
dorsally over the second metatarsal head to identify segment ends. Limb segment motion was tracked by three markers rigidly fixed to thermoplastic shells, which were wrapped securely to the thighs and shanks. Subjects walked with comfortable shoes on a $10 \mathrm{~m}$ walkway at a self-selected walking speed for a minimum of five trials. Subjects were tested without walking aids, such as walkers or canes, and without ankle-foot-orthoses (AFOs), when possible. The increased lateral stability provided by assistive devices may alter the relationships between compensatory motions and strength and coordination measures. Additionally, from a function perspective, subjects do not use walking aids at all times.

Single joint isometric strength of the paretic knee and ankle joints was measured in a seated posture with the hip at $90^{\circ}$ using a Biodex ${ }^{\circledR}$ System 3 (Biodex Medical Systems; Shirley, NY), previously described (Lewek et al., 2006). The knee was placed in $0^{\circ}$ (i.e., full extension) to test ankle strength in dorsiflexion (ankle at $30^{\circ}$ plantarflexion) and plantarflexion (ankle in neutral). Knee flexion strength was collected with the knee flexed to $30^{\circ}$ while knee extension strength was tested in $60^{\circ}$ knee flexion. Subjects produced three maximum voluntary isometric contractions (MVIC) for each target torque, with at least 1 min rest between attempts. While not identical to toeoff gait angles, data collected from similar postures have been related to gait speed (Bohannon and Andrews, 1990; Hsu et al., 2003).

Isometric hip strength was measured in a standing posture mimicking the limb configuration of toeoff $\left(15^{\circ}\right.$ hip extension and $45^{\circ}$ knee flexion) using an experimental paradigm described previously (Cruz and Dhaher, 2008). The pelvis and paretic limb were secured to an exoskeleton (Lokomat, Switzerland) instrumented with three 6-degree-of-freedom load cells (JR3, Woodland, CA). The joints of the exoskeleton were locked at toeoff using mechanical stops. Subjects were given three opportunities to produce maximal hip flexion/extension and abduction/adduction torques while receiving visual feedback regarding the direction and magnitude of the torque produced (1000 Hz sampling rate).

Abnormal coordination between the musculature of the paretic hip and knee joints was also assessed in the standing isometric hip posture described above. Subjects used real-time feedback to produce abduction and adduction torques at the hip joint, equivalent to $50 \%$ of the maximum values, while flexion/extension knee torques were recorded simultaneously without feedback. The hip/knee torque coupling was defined as the ratio of knee flexion/ extension torque to hip abduction/adduction torque. Our previous work has shown that stroke subjects typically couple knee extension with hip adduction and knee flexion with hip abduction (defined as positive), while control subjects do not produce torque coupling as a group (Cruz and Dhaher, 2008).

\subsection{Data analysis}

Marker trajectories obtained during the gait analysis were identified and low-pass filtered (6 $\mathrm{Hz}$ ) to track the 3D motion of the pelvis and lower limb segments using EvaRT software (Motion Analysis Corp., Los Angeles, CA). The relative positions and inter-segmental joint angles were calculated using a rigid body analysis (Grood and Suntay, 1983) and normalized to one gait cycle using OrthoTrak 6.2.4. Gait speed was calculated using the time derivative of the sacral marker position in the direction of forward progress.

Because the mechanical state of the lower limb at the initiation of swing influences swing phase dynamics (Goldberg et al., 2003), we focused our investigation of gait dysfunction on the toeoff posture. Specifically, the kinematic measures of interest were pelvic obliquity (i.e., the frontal plane angle of the pelvis) and abduction/adduction hip angle (i.e., the frontal plane angle of the femur with respect to the pelvis) at toeoff. The first measure was derived from previous work which defined hip hiking as excessive frontal plane pelvic angle 
(Kerrigan et al., 2000). The second measure was chosen because hip frontal plane angle is generally accepted as a contributor to circumduction, although discrepancies in the definition exist (Kerrigan et al., 2000; Perry, 1992). In addition, pelvic obliquity and hip abduction/adduction velocities were calculated by taking the first derivative of the respective angles. Given our interest in the relative compensation of these velocity measures, we have operationally defined the compensatory kinematic measures for the Stroke group as deviations from the mean Control group value of pelvic obliquity velocity and hip abduction/adduction velocity at toeoff.

To quantify the isometric torques, load cell signals were filtered off-line using a zero-phase, low-pass, fourth-order Butterworth filter with a $50 \mathrm{~Hz}$ cutoff frequency. The hip and knee joint torques were calculated from the load cell signals using static equilibrium equations. To account for rotational differences of the flexed limb between planes, the frontal and sagittal plane torques were normalized by their respective moment of inertia (Zatsiorsky and Seluyanov, 1985). For consistency, the knee and ankle torques were also normalized by the moment of inertia of the shank plus foot and foot, respectively. Peak joint torques were defined as the maximum torque value maintained for at least $200 \mathrm{~ms}$.

Gait speed and frontal plane kinematics were compared between the Stroke and Control groups using independent samples $t$-tests (alpha $=.05$ and $p<0.05$ ). Distributions of all variables were tested for normalcy (NCSS 2004, Kaysville, UT). Stepwise linear regression models were used to determine the most significant predictors of gait speed and compensatory hip and pelvis velocity measures from the pool of single joint strength (paretic hip abduction, adduction, flexion, and extension, knee flexion and extension, and ankle plantarflexion and dorsiflexion) and multi-joint synergy measures (knee flexion-to-hip abduction ratio and knee extension-to-hip adduction ratio). Then, multiple linear regression models were created to relate these variables to the dependent variables (Tamhane and Dunlop, 2000). To assess the relative predictive power of the independent variables, the data were first standardized (centered and scaled by the standard deviation (Tamhane and Dunlop, 2000)). This procedure prevents bias due to effect size by the independent variables. Inter-subject differences were modeled by treating subjects as random effects, each with their own error structure. Using a least square fit algorithm with no interactions (i.e., no cross product terms), linear combinations of variables were created that minimized the error in the prediction of the dependent variable. The best model was deemed to have only significant factors and a good fit reflected by the $r^{2}$. The regression coefficients reflect the contribution of the predictor variables on gait speed and compensatory hip/pelvis kinematics. Significance for the models was set at $p=0.05$. Power was tested at the alpha $=$ 0.05 level. Normal probability plots for the residuals were checked to ensure that the assumptions about the models were met. Additionally, correlation between the outcome variables was tested for covariance.

\section{Results}

Gait analysis was performed on 18 subjects with stroke and 8 control subjects (see Table 1). The groups significantly differed in pelvic obliquity angle at toeoff ( $p=0.005$, Fig. 1$)$, but not in frontal plane hip angle $(p=0.44)$. The Stroke group also walked with a significantly $(p=0.002)$ slower gait speed $(0.76 \mathrm{~m} / \mathrm{s}(\mathrm{SD} 0.19))$ compared to the Control group $(1.26 \mathrm{~m} / \mathrm{s}$ (SD 0.30), see Table 2). The hip strength and multi-joint coupling values were collected from all 18 subjects in the Stroke group. Due to scheduling difficulties, knee and ankle strength data were not collected from two subjects (see Table 3).

Stepwise regression analysis selected four variables to best estimate gait speed. In decreasing order of influence, the model $(n=18)$ included the ratio of knee extension-to-hip 
adduction torque, the ratio of knee flexion-to-hip abduction torque, hip extension strength, and hip abduction strength (see Table 4A). This model was statistically significant ( $p=$ 0.003 ) with high power (0.956). The remaining six variables (paretic hip adduction and flexion, knee flexion and extension, and ankle dorsiflexion and plantarflexion strength) did not statistically improve the $r^{2}$ value for the model. Based on the model, reduced gait speed was associated with greater multi-joint coupling and paretic hip abduction strength. On the contrary, greater gait speed was associated with greater paretic hip extension torque.

Four of the 10 variables were significantly related to deviations in pelvic obliquity velocity $(n=15 ; p<0.001)$. In decreasing order of influence, the variables were hip extension strength, the ratio of knee extension-to-hip adduction torque, knee flexion strength, and hip flexion strength, see Table 4B. The multiple linear regression model revealed that abnormal coupling was associated with an increased deviation from control values, while greater strength in the paretic hip extensors and flexors, and knee flexors were all associated with reduced deviations in pelvic obliquity velocity from control values. The regression algorithms were unable to find a relationship between the strength variables and frontal plane hip velocity deviations at toeoff in the Stroke group. Gait speed and deviations in pelvic obliquity velocity were not significantly correlated $(p>0.05)$, justifying the separate regression analyses.

\section{Discussion}

This investigation examined associations among lower limb strength and coordination impairments and walking abnormalities in individuals post-stroke. Specifically, multiple linear regression analysis revealed that paretic hip extension strength was positively related to gait speed, while paretic hip abduction strength and the multi-joint coupling of hip frontal plane and knee sagittal plane torques were negatively related to gait speed. Our analysis also showed that coupling between hip adduction and knee extension torque was positively associated with pelvic obliquity velocity deviations. On the contrary, paretic hip flexion and extension strength, and knee flexion strength were negatively associated with pelvic obliquity velocity deviations. These findings indicate that different, yet overlapping sets of impairments were associated with gait outcomes. Delineation of the relative effects of impaired strength and across-joint coordination on gait speed and compensatory kinematic patterns may motivate targeted interventions to differentially restore these gait characteristics.

Prior studies have reported a positive association between isokinetic hip flexion strength and overground walking speed using both single (Hsu et al., 2003; Kim and Eng, 2003; Nadeau et al., 1999a) and multiple linear regression techniques (Kim and Eng, 2003; Nadeau et al., 1999a). Hip flexion strength tested in an isometric, upright, and functional position, however, was not associated with gait speed. While differences in experimental paradigms may have contributed to the conflicting outcomes, isometric and isokinetic torques are similar when matched for posture (Babault et al., 2001). Although hip flexion strength was not related to gait speed, our data did reveal significant relationships between hip and knee flexion torques and pelvic obliquity velocity deviations. Specifically, a decrease in both hip and knee flexion torques was associated with an increase in pelvic obliquity velocity deviation, which may be used to facilitate swing phase toe clearance in the presence of reduced hip and knee flexion angle.

A key finding of this investigation is the observed associations of hip extension strength to both gait speed and pelvic obliquity in the chronic stroke population. Recent musculoskeletal modeling studies have shown that the hip extensor gluteus maximus, in addition to the ankle plantarflexors gastrocnemius and soleus, contributes to the forward 
acceleration of the trunk during overground walking (Neptune et al., 2004; Zajac et al., 2003). Given that ankle plantarflexor strength is frequently impaired post-stroke (Neckel et al., 2006), we propose that subjects may generate forward propulsion by employing a hip extension torque strategy during stance (Neptune et al., 2004). Focal neural blocks to selectively target either ankle plantarflexors or hip extensors may delineate the differential contribution of each muscle group to gait velocity. This knowledge may guide targeted strength training to increase gait velocity post-stroke.

Previous investigations have noted that the energetic demands of walking are increased following stroke (Zamparo et al., 1995) and may be associated with increased need for lateral stabilization during walking (Dean et al., 2007). Given that assistive devices are often used to provide lateral stabilization, we performed the gait analysis without assistive devices, to determine the compensations required in the presence of muscle weakness and impaired coordination. Our results demonstrate the importance of hip frontal plane (abduction/adduction) torques during walking, and thus expand prior work by suggesting that lateral stabilization is maintained, in part, by hip abductor and adductor muscles. This argument is consistent with findings from dynamic walking models that suggest a frontal plane hip torque may maintain stability during gait (Kuo, 1999). Given that the hip flexion/ extension angle has little effect on the frontal plane moment arms of the hip musculature, it is reasonable to suggest that similar abduction torque strength would be found at toeoff and stance postures. As a result of the increased energetic demand for frontal plane stabilization, stability control may come at the expense of speed in the stroke population (Chen et al., 2003). Therefore, therapeutic interventions aimed at improving speed should take into account the potential effects on lateral stability.

In the current study, we observed functional implications of multi-joint (knee/hip) torque coupling. Kinematically, coupling of hip adduction and knee extension torque would increase the effective leg length thereby necessitating compensatory hip hiking for toe clearance. On the contrary, hip abduction and knee flexion coupling failed to associate with pelvic obliquity velocity, presumably because knee flexion and hip abduction facilitate toe clearance negating the need for hip hiking. These findings extend the body of evidence relating functional movement deficits to abnormal multi-joint torque synergies (Beer et al., 2000; Dewald and Beer, 2001). Furthermore, motor training that targets joint actions against synergistic coupling may be useful in improving both gait speed and compensatory kinematics (Ellis et al., 2005).

The choice of preferred gait speed rather than maximal gait speed for the gait performance metric was based on the desire to understand the more frequently displayed gait dysfunction. While strength is not the only contributor to preferred gait speed, it is also one factor that may be altered through rehabilitation (Patten et al., 2004).

The multi-factorial findings suggest that therapies directed at multiple joints may provide more beneficial results than those aimed at resolving single joint impairments. The different yet overlapping groups of significant impairments suggest that gait speed and some compensatory kinematics may be manifestations of different motor impairments post-stroke. Targeted rehabilitation paradigms that increase strength at multiple joints and reduce acrossjoint torque coupling may, therefore, selectively increase gait speed or restore normal frontal plane kinematics. While this investigation has focused on the chronic stroke population, the development of similar associations in the acute phase may be useful for the allocation of resources to maximize gait recovery. 


\section{Acknowledgments}

We would like to thank Preeti Nair, PT PhD for editing this manuscript. We would also like to thank Jennifer Moore, MPT, Heidi Roth, MSPT, and Winsean Lin, MS for assistance with data collection and analysis.

Sources of Funding. This research was made possible by funding from the Searle Fund, the American Heart Association (Predoctoral Fellowship no. 0610062Z) and by the US Department of Education, National Institute on Disability and Rehabilitation Research (Field Initiated Grant no. H133A990008).

\section{References}

Babault N, Pousson M, Ballay Y, Van Hoecke J. Activation of human quadriceps femoris during isometric, concentric, and eccentric contractions. J. Appl. Physiol. 2001; 91:2628-2634. [PubMed: 11717228]

Beer RF, Dewald JP, Rymer WZ. Deficits in the coordination of multijoint arm movements in patients with hemiparesis: evidence for disturbed control of limb dynamics. Exp. Brain Res. 2000; 131:305319. [PubMed: 10789946]

Bohannon RW, Andrews AW. Correlation of knee extensor muscle torque and spasticity with gait speed in patients with stroke. Arch. Phys. Med. Rehabil. 1990; 71:330-333. [PubMed: 2327887]

Chen CL, Chen HC, Tang SF, Wu CY, Cheng PT, Hong WH. Gait performance with compensatory adaptations in stroke patients with different degrees of motor recovery. Am. J. Phys. Med. Rehabil. 2003; 82:925-935. [PubMed: 14627929]

Chen G, Patten C, Kothari DH, Zajac FE. Gait differences between individuals with post-stroke hemiparesis and non-disabled controls at matched speeds. Gait Posture. 2005; 22:51-56. [PubMed: 15996592]

Cruz T, Dhaher Y. Evidence of abnormal lower limb torque synergies after stroke: an isometric study. Stroke. 2008; 39:139-147. [PubMed: 18063824]

Dean JC, Alexander NB, Kuo AD. The effect of lateral stabilization on walking in young and old adults. IEEE Trans. Biomed. Eng. 2007; 54:1919-1926. [PubMed: 18018687]

Dewald JP, Beer RF. Abnormal joint torque patterns in the paretic upper limb of subjects with hemiparesis. Muscle Nerve. 2001; 24:273-283. [PubMed: 11180211]

Ellis MD, Holubar BG, Acosta AM, Beer RF, Dewald JP. Modifiability of abnormal isometric elbow and shoulder joint torque coupling after stroke. Muscle Nerve. 2005; 32:170-178. [PubMed: 15880629]

Goldberg SR, Ounpuu S, Delp SL. The importance of swing-phase initial conditions in stiff-knee gait. J. Biomech. 2003; 36:1111-1116. [PubMed: 12831736]

Grood ES, Suntay WJ. A joint coordinate system for the clinical description of three-dimensional motions: application to the knee. J. Biomech. Eng. 1983; 105:136-144. [PubMed: 6865355]

Hsu AL, Tang PF, Jan MH. Analysis of impairments influencing gait velocity and asymmetry of hemiplegic patients after mild to moderate stroke. Arch. Phys. Med. Rehabil. 2003; 84:1185-1193. [PubMed: 12917858]

Kerrigan DC, Frates EP, Rogan S, Riley PO. Hip hiking and circumduction: quantitative definitions. Am. J. Phys. Med. Rehabil. 2000; 79:247-252. [PubMed: 10821310]

Kim CM, Eng JJ. The relationship of lower-extremity muscle torque to locomotor performance in people with stroke. Phys. Ther. 2003; 83:49-57. [PubMed: 12495412]

Kuo A. Stabilization of lateral motion in passive dynamic walking. Int. J. Robotics Res. 1999; 18:917930.

Lewek MD, Schmit BD, Hornby TG, Dhaher YY. Hip joint position modulates volitional knee extensor muscle activity after stroke. Muscle Nerve. 2006; 34:767-774. [PubMed: 16967491]

Lin PY, Yang YR, Cheng SJ, Wang RY. The relation between ankle impairments and gait velocity and symmetry in people with stroke. Arch. Phys. Med. Rehabil. 2006; 87:562-568. [PubMed: 16571398]

Nadeau S, Arsenault AB, Gravel D, Bourbonnais D. Analysis of the clinical factors determining natural and maximal gait speeds in adults with a stroke. Am. J. Phys. Med. Rehabil. 1999a; 78:123-130. [PubMed: 10088586] 
Nadeau S, Gravel D, Arsenault AB, Bourbonnais D. Plantarflexor weakness as a limiting factor of gait speed in stroke subjects and the compensating role of hip flexors. Clin. Biomech. (Bristol, Avon). 1999b; 14:125-135.

Neckel ND, Pelliccio M, Nichols D, Hidler JM. Quantification of functional weakness and abnormal synergy patterns in the lower limb of individuals with chronic stroke. J. Neuroengineering Rehabil. 2006; 3:17.

Neptune RR, Zajac FE, Kautz SA. Muscle force redistributes segmental power for body progression during walking. Gait Posture. 2004; 19:194-205. [PubMed: 15013508]

Patten C, Lexell J, Brown HE. Weakness and strength training in persons with poststroke hemiplegia: Rationale, method, and efficacy. J. Rehabil. Res. Dev. 2004; 41:293-312. [PubMed: 15543447]

Perry, J. Gait Analysis: Normal and Pathological Function. SLACK Thorofare; NJ: 1992.

Perry J, Garrett M, Gronley JK, Mulroy SJ. Classification of walking handicap in the stroke population. Stroke. 1995; 26:982-989. [PubMed: 7762050]

Tamhane, A.; Dunlop, DD. Statistics and Data Analysis: From Elementary to Intermediate. Prentice Hall, Upper Saddle River; NJ: 2000.

Zajac FE, Neptune RR, Kautz SA. Biomechanics and muscle coordination of human walking: part II: lessons from dynamical simulations and clinical implications. Gait Posture. 2003; 17:1-17. [PubMed: 12535721]

Zamparo P, Francescato MP, De Luca G, Lovati L, di Prampero PE. The energy cost of level walking in patients with hemiplegia. Scand. J. Med. Sci. Sports. 1995; 5:348-352. [PubMed: 8775719]

Zatsiorsky, V.; Seluyanov, V. Estimation of the mass and inertia characteristics of the human body by means of the best predictive regression equations.. In: Winter, D.; Norman, R.; Wells, R.; Hayes, K.; Patla, A., editors. Biomechanics IX-B. Human Kinetics, Champaign; IL: 1985. p. 233-239. 


\section{Pelvic Obliquity Angle}

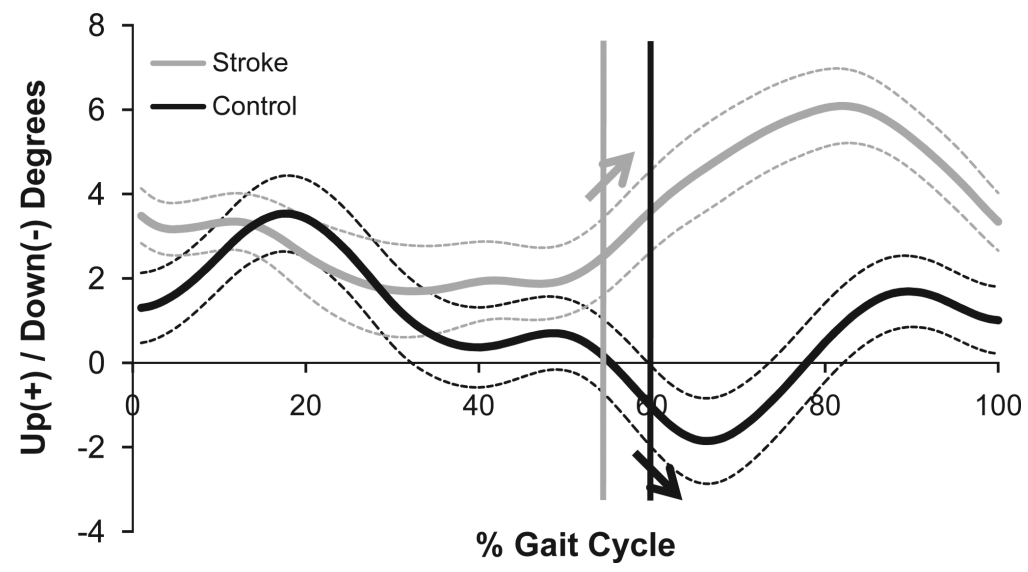

Fig. 1.

Mean $( \pm \mathrm{SE})$ pelvic obliquity angle for Stroke (grey) and Control (black) groups. Vertical lines indicate respective toeoffs and arrows indicate pelvic obliquity velocity. 


\section{Table 1}

Subject demographics.

\begin{tabular}{lll}
\hline & Stoke $(\boldsymbol{n}=\mathbf{1 8})$ & Control $(\boldsymbol{n}=\mathbf{8})$ \\
\hline Age & $54.8(9.9)$ & $51.8(9.4)$ \\
Time post stroke (mos) & $78.9(60.5)$ & NA \\
Sex & 12 Males & 5 \\
Hemiparetic side & 11 Right & NA \\
Height (m) & $1.72(0.09)$ & $1.74(0.09)$ \\
Mass $(\mathrm{kg})$ & $78.6(17.5)$ & $74.0(11.1)$ \\
AFO & 4 AFO & NA \\
\hline
\end{tabular}

Mean (Standard Deviation) 
Table 3

Stroke group isometric strength.

\begin{tabular}{|c|c|c|}
\hline & Mean (SE) (Nm/kg m²) & Range $\left(\mathrm{Nm} / \mathrm{kg} \mathrm{m}^{2}\right)$ \\
\hline Paretic hip flexion & $10.76(1.38)$ & $0-21.93$ \\
\hline Paretic hip extension & $16.07(1.95)$ & $0-38.7$ \\
\hline Paretic hip abduction & $13.92(1.74)$ & $1.66-35.84$ \\
\hline Paretic hip adduction & $12.36(1.82)$ & $0-29.92$ \\
\hline Paretic knee flexion & $32.78(5.26)$ & $6.86-70.81$ \\
\hline Paretic knee extension & $77.42(9.14)$ & $9.67-142.40$ \\
\hline Paretic dorsiflexion & $534.06(74.71)$ & $0-1356.76$ \\
\hline Paretic plantarflexion & $1746.92(221.74)$ & $500.81-3827.57$ \\
\hline Multi-joint coupling — ratio of paretic knee extension to hip adduction & $1.2(.34)$ & -1.05 to 6.39 \\
\hline Multi-joint coupling_-ratio of paretic knee flexion to hip abduction & $.29(.11)$ & -.59 to 1.04 \\
\hline
\end{tabular}

Single joint strength and multi-joint torque coupling measures normalized to moment of inertia.

Note: The large ankle strength values are a result of the relatively small inertia of the foot. 
Table 4

\begin{tabular}{|c|c|c|c|c|}
\hline Source & Coefficient & $R^{2}$ & Probability level & Power 5\% \\
\hline \multicolumn{5}{|c|}{ A-ANOVA for multiple regression model for gait speed } \\
\hline Hip abduction & -0.44 & & 0.012 & .767 \\
\hline Hip extension $^{a}$ & 0.46 & & 0.024 & .651 \\
\hline Knee flexion/Hip abduction ratio & -0.49 & & 0.024 & .652 \\
\hline Knee extention/Hip adduction ratio ${ }^{a}$ & -0.63 & & 0.007 & .831 \\
\hline Model & & .68 & 0.003 & .956 \\
\hline \multicolumn{5}{|c|}{ B-ANOVA for multiple regression model for pelvic obliquity compensation } \\
\hline Hip flexion & -0.35 & & 0.002 & .966 \\
\hline Hip extension ${ }^{a}$ & -0.88 & & 0.000 & 1.00 \\
\hline Knee flexion & -0.38 & & 0.001 & .993 \\
\hline Knee extension/Hip adduction ratio ${ }^{a}$ & 0.56 & & 0.000 & 1.00 \\
\hline Model & & .957 & 0.000 & 1.00 \\
\hline
\end{tabular}

Only statistically significant independent variables shown.

Independent variable common to both models. 